\title{
An Algebraic Spin and Statistics Theorem
}

\author{
Daniele Guido $^{1 *}$ and Roberto Longo ${ }^{1,2 *}$ \\ (1) Dipartimento di Matematica, Università di Roma "Tor Vergata" \\ via della Ricerca Scientifica, I-00133 Roma, Italia. \\ $\left({ }^{2}\right)$ Centro Linceo Interdisciplinare, Accademia Nazionale dei Lincei \\ via della Lungara 10, I-00165 Roma, Italia \\ Dedicated to Hans Borchers on the occasion of his seventieth birthday
}

To appear in Commun. Math. Phys. (1995).

\begin{abstract}
A spin-statistics theorem and a PCT theorem are obtained in the context of the superselection sectors in Quantum Field Theory on a 4-dimensional space-time. Our main assumption is the requirement that the modular groups of the von Neumann algebras of local observables associated with wedge regions act geometrically as pure Lorentz transformations. Such a property, satisfied by the local algebras generated by Wightman fields because of the Bisognano-Wichmann theorem, is regarded as a natural primitive assumption.
\end{abstract}

* Supported in part by MURST and CNR-GNAFA. E-mail: guido@mat.utovrm.it, longo@mat.utovrm.it 


\section{Introduction}

In this paper we shall reconsider from an intrinsic point of view two well known fascinating theorems in Quantum Field Theory: the PCT theorem and the Spin and Statistics theorem.

Both of them have a long history, see $[28,43]$. The spin and statistics theorem first appeared in the context of free fields in the work of Fierz [20] and Pauli [37]: one cannot second quantize particles with integer spin by anticommuting fields, i.e. fields obeying Fermi statistics, nor particles with half-integer spin by local fields, i.e. fields obeying Bose statistics.

The PCT theorem originated in [35] as a relation between the existence of the space-inversion symmetry $\mathrm{P}$ and the existence of the product of the charge and the time-inversion symmetry CT. Pauli proved in [38] that PCT is always a symmetry of Lorentz invariant field equations.

It was a success of the Wightman axiomatic approach [46] to establish model independent results: the connection between spin and statistics was obtained by Burgoyne [13], see also [36,14], and a PCT theorem by Jost [29], see also [19, 39], both relying on the general holomorphic properties of the $n$-point functions. A spin and statistics theorem in the algebraic approach [27], see also [5], was later given by Epstein [21] and has a version for (Doplicher-Haag-Roberts) DHR superselection sectors [16] and for more general topological charges [10].

All these approaches heavily rely on arguments of analytic continuation, whose nature give some mysterious effectiveness to the results. Moreover they make use of certain detailed structures, either because they deal with Wightman tempered distributions or because they treat the case of finite mass degeneracy, where a supeselection sector has to contain only finitely many particles of the same type and all of them are assumed to have strictly positive mass.

The approach to Quantum Field Theory by local observable algebras [27] suggests however that a PCT symmetry and spin-statistics correspondence should be intrinsically associated with the net of local algebras and manifest itself as the consequence of the locality principle ${ }^{1}$.

From the mathematical point of view the spin-statistics correspondence is a relation between two quantities of different nature, the univalence and the statistics phase, and one is led to tie up these concepts on general grounds, somehow in the spirit of an index theorem.

We shall establish both a PCT and a spin-statistics theorem in the following general context.

Let $\mathcal{O} \rightarrow \mathcal{A}(\mathcal{O})$ be a net of von Neumann algebras on a Hilbert space $\mathcal{H}$, i.e. an inclusion preserving association between regions $\mathcal{O}$ in the four-dimensional Minkowski space and von Neumann algebras of local observables, that we assume here to be irreducible.

We make the following assumptions.

Locality. If $\mathcal{O}_{1}$ and $\mathcal{O}_{2}$ are space-like separated regions, then $\mathcal{A}\left(\mathcal{O}_{1}\right)$ and $\mathcal{A}\left(\mathcal{O}_{2}\right)$ commute elementwise.

Modular covariance. There is a vector $\Omega \in \mathcal{H}$, the vacuum vector, cyclic for the algebras $\mathcal{A}(W)$ associated with all wedge region $W$ in the Minkowski space, such that

$$
\Delta_{W}^{i t} \mathcal{A}(\mathcal{O}) \Delta_{W}^{-i t}=\mathcal{A}\left(\Lambda_{W}(t) \mathcal{O}\right), \quad t \in \mathbf{R}
$$

where $\mathcal{O}$ is any region, $\Delta$ is the Tomita-Takesaki modular operator [44,41] associated with $(\mathcal{A}(W), \Omega)$ and $\Lambda_{W}$ is the one-parameter rescaled group of pure Lorentz transformations preserving $W$.

Reeh-Schlieder property. The vacuum vector $\Omega \in \mathcal{H}$ is also cyclic for the algebras $\mathcal{A}(\mathcal{S})$ associated with all space-like cones $\mathcal{S}$.

Locality is the well-known expression of Einstein causality and we do not dwell on it. The Reeh Schlieder property is known to hold for the vacuum vector in a Poincaré covariant theory as a consequence of the positivity of the energy and the weak additivity assumption for the local algebras [7].

Modular covariance needs however further comments. Postponing for a while the justification for such an assumption, we recall that this entails the net to be covariant with respect to the universal covering $\widetilde{\mathcal{P}}_{+}^{\uparrow}$

1 The spin-statistics relation depends on sharp locality. In the second quantization of Bose particles by anti-commuting fields, microscopic causality is still asymptotically present and its violation is sizeable only at distances comparable to the Compton wave length [4]. This is perhaps an indication that the spin-statistics relation might be different in contexts like Quantum Gravity where a sharp causality principle does not occur. 
of the Poincaré group $\mathcal{P}_{+}^{\uparrow}$, with positive energy [9]. Indeed we shall prove here that it is actually covariant with respect to $\mathcal{P}_{+}^{\uparrow}$ as a special case of our general Spin and Statistics theorem and taking to completion our previous work. Therefore modular covariance is a way to intrinsically encode the Poincaré covariance property in the net structure, providing a canonical representation of the Poincaré group $\mathcal{P}_{+}^{\uparrow}$ (cf. also [12]).

Let now $\rho$ be a superselection sector of $\mathcal{A}$ in the sense of Doplicher-Haag-Roberts [15] or more generally of Buchholz-Fredenhagen [11].

An index-statistics relation [34] shows that

$$
\operatorname{Ind}(\rho)=d(\rho)^{2}
$$

where $\operatorname{Ind}(\rho)$ is the Jones index of $\rho$ and $d(\rho)$ is the DHR statistical dimension, namely

$$
d(\rho)=\left|\lambda_{\rho}\right|^{-1}
$$

where the statistical parameter $\lambda_{\rho} \in \mathbf{R}$ classifies the statistics in $3+1$ space-time dimensions [15].

Therefore the index is an intrinsic quantity that determines the statistics up to the Fermi-Bose alternative, i.e. the sign of $\lambda_{\rho}$.

On the other hand, the Poincaré representation in the vacuum sector being fixed by modular covariance, the representation of $\widetilde{\mathcal{P}}_{+}^{\uparrow}$ associated with a covariant irreducible sector $\rho$ is uniquely determined, therefore the univalence (integer or half-integer spin alternative) is intrinsically associated with $\rho$. Since $\rho$ is automatically $\widetilde{\mathcal{P}}_{+}^{\uparrow}$-covariant if $d(\rho)<\infty$ (assuming a regularity property for the net [22]), it is natural to expect a general algebraic Spin and Statistics theorem connecting these two intrinsic quantities for any sector with finite statistics.

Our result in this respect will in fact show that on these general grounds

$$
\operatorname{sign}\left(\lambda_{\rho}\right)=U_{\rho}(2 \pi)
$$

where $U_{\rho}$ is the representation of $\widetilde{\mathcal{P}}_{+}^{\uparrow}$ in the sector $\rho$ and $U_{\rho}(2 \pi)$ denotes the corresponding rotation by $2 \pi$.

Modular covariance also implies that the anti-unitary involution $\Theta$, definable by the modular theory according to the Bisognano-Wichmann prescription [3], implements a complete space-time reflection. As shown in [22], this entails that $\Theta$ intertwines a sector with its conjugate. We therefore obtain a PCT symmetry.

We come now back to the origin of the modular covariance property. Its main justification certainly comes from the Bisognano-Wichmann theorem [2,3], to the effect that this property holds if the local algebras are constructed from Wightman fields.

An algebraic version of the Bisognano-Wichmann theorem does not exist yet, except in the case of conformal theories where it holds in full generality [8]. However a theorem of Borchers [6] shows part of the geometric properties of the modular group for wedge regions to be always present and in particular every $1+1$ dimensional Poincaré covariant net satisfying essential duality has the modular covariance property.

At the present time no counter-example to modular covariance is known to exist within Poincaré covariant theories (see however $[33,48]$ ). There are nevertheless counter-examples to the spin-statistics theorem [42]: these are constructed by infinite multiplicity fields where the Poincaré group representation is not unique. It turns out that the wrong connection between spin and statistics depends on the wrong choice of the Poincare group representation, while our canonical choice for the latter has the desired properties. We remark that an intrinsic way to eliminate pathological examples of the above kind comes by requiring the split property [17]; this indeed implies the uniqueness of the Poincaré group representation [8] and we propose it as a natural candidate for a derivation of the modular covariance property by first principles.

On the physical side modular covariance manifests an interesting analogy with the Unruh effect [45] and with the Hawking black hole thermal radiation [26], as first noticed by Sewell [40]. We sketch the essential ideas, see also [24]. As is known the modular group of a von Neumann algebra with respect to a given state is characterized by the Kubo-Martin-Schwinger condition [44] and, on the other hand, the KMS condition is peculiar of thermal equilibrium states in Statistical Mechanics [25]. By the Bisognano-Wichmann theorem the boosts satify the KMS condition with respect to the vacuum, as automorphisms of the von Neumann algebra of the corresponding wedge $W$ and, on the other hand, the orbits of the boosts are the trajectories 
of a uniformly accelerated motion for which the "Rindler universe" $W$ is a natural horizon; the equivalence principle in Relativity Theory then allows an interpretation of the thermal outcome as a gravitational effect. On this basis Haag has proposed long ago to derive the Bisognano-Wichmann theorem.

The role of the modular covariance assumption may be also understood by its consequences. Among other things, it implies the positivity of the energy for the constructed Poincaré group representation $[47,9]$. As is known the positivity of the energy is lost on a curved space-time, and the modular covariance seems to be the appropriate substitute in this case. Moreover, as already mentioned, it gives rise to the KMS condition, namely an analytic continuation property. It turns out that this analytic aspect of the modular covariance assumption incorporates all the holomorphic properties present in Quantum Field Theory. But, as a matter of fact, the modular group is an algebraic object, a manifestation of the *-operation, thus providing us with an algebraic approach to our problems.

We pass now to a description of the methods of our work. This paper relies on the modular theory of Tomita and Takesaki and on an analysis by the unitary representations of $\operatorname{SL}(2, \mathbf{R})$.

We shall find a key relation arising from the comparison of the modular groups of different algebras, and we shall regard it as an identity concerning operators in the space of a representation of $\operatorname{SL}(2, \mathbf{R})$, because of the well known fact that the $2+1$ dimensional Lorentz group is isomorphic to $\mathrm{SL}(2, \mathbf{R}) /\{1,-1\}$. Section 1 contains the proof of this identity by the Mackey machine of the induced representations (e.g. [32]) and a free field verification.

In Sect. 2 the PCT theorem and the Spin and Statistics relation are proven in the context of the field algebras ${ }^{2}$ where the formalism is close to the classical formulation. Then, in Sect. 3, we obtain our result in the context of local observables. This is done by rephrasing the statements in terms of the Doplicher-Roberts field algebra [18]. This last step has certain pedagogical advantages, but has to be avoided in order to extend our work to more general settings where the field algebra does not exist.

In forthcoming papers [23] we shall indeed provide a more intrinsic approach in terms of local observables only, that will cover low dimensional and conformal theories in particular. The general picture will be clarified by examples.

\section{An Identity for Operators Associated with Representations of $\operatorname{SL}(2, \mathbf{R})$}

Let us consider two one-parameter subgroups of $\mathrm{SL}(2, \mathbf{R})$

$$
\mu(t) \equiv\left(\begin{array}{cc}
\cosh \pi t & -\sinh \pi t \\
-\sinh \pi t & \cosh \pi t
\end{array}\right), \quad \nu(t) \equiv\left(\begin{array}{cc}
e^{-\pi t} & 0 \\
0 & e^{\pi t}
\end{array}\right) .
$$

If $U$ is a unitary representation of $\operatorname{SL}(2, \mathbf{R})$ on a Hilbert space $\mathcal{H}$ we look at the corresponding selfadjoint infinitesimal generators

$$
\begin{aligned}
& H=H_{U}=\left.i \frac{d}{d t} U(\mu(t))\right|_{t=0}, \\
& K=K_{U}=\left.i \frac{d}{d t} U(\nu(t))\right|_{t=0} .
\end{aligned}
$$

We shall denote by $\mathbf{G}$ the group $\operatorname{PSL}(2, \mathbf{R})$ given by the quotient of $\operatorname{SL}(2, \mathbf{R})$ by its center $\{-1,1\}$ and by $\widetilde{\mathbf{G}}$ the universal covering of $\mathbf{G}$, which is of course the universal covering of $\operatorname{SL}(2, \mathbf{R})$ too. We take the same definition for $H$ and $K$ in the case of a unitary representation $U$ of the universal covering group $\widetilde{\mathbf{G}}$.

We will consider the following properties for a representation $U$ of $\widetilde{\mathbf{G}}$ :

(i) The operator $T_{t} \equiv e^{\frac{1}{2} K} e^{i t H} e^{-\frac{1}{2} K}$ is densely defined for all $t \in \mathbf{R}$.

(ii) $T_{t}$ is densely defined and

$$
e^{\frac{1}{2} K} e^{i t H} e^{-\frac{1}{2} K} \subset e^{-i t H}
$$

where the symbol $\subset$ denotes the extension of operators. These properties refer to a representation $U$, but we omit the symbol $U$ when no confusion arises.

${ }^{2}$ We have recently been informed by Kuckert of an independent complementary analysis based on assumptions of weak geometric type for the modular conjugation [31]. 
1.1. Theorem. (a) Property (ii) holds for the regular representation $\lambda$ of $\mathbf{G}$.

(b) Property (i) implies Property (ii).

In order to prove this theorem, we first observe that it is enough to check Property $(i i)$ on dense sets of vectors, not necessarily on the full domain of $T_{t}$.

1.2. Lemma. Let us assume that, for each real $t$, there is a dense subset $\mathcal{D}_{t}$ of the domain of $T_{t}$ such that $\left.T_{t}\right|_{\mathcal{D}_{t}} \subset e^{-i t H}$. Then Property (ii) holds for the given representation.

Proof. Note first that the matrix $\left(\begin{array}{cc}0 & 1 \\ -1 & 0\end{array}\right) \in \mathrm{SL}(2, \mathbf{R})$ conjugates $\mu(t)$ with $\mu(-t)$ and $\nu(t)$ with $\nu(-t)$ therefore the assumption of the lemma remains true if we replace $K$ with $-K$ and $H$ with $-H$, in particular $e^{-\frac{1}{2} K} e^{i t H} e^{\frac{1}{2} K}$ is densely defined. Now

$$
T_{t}^{*} \supset e^{-\frac{1}{2} K} e^{-i t H} e^{\frac{1}{2} K}
$$

therefore $T_{t}^{*}$ is densely defined and $T_{t}$ is closable. Since $T_{t} \xi=e^{-i t H} \xi$ for all $\xi$ in a dense set and $e^{-i t H}$ is bounded, the equality $T_{t} \xi=e^{-i t H} \xi$ must hold for all $\xi$ in the domain of $T_{t}$.

1.3. Corollary. Let $U_{1}, U_{2}$ and $U$ be unitary representations of $\widetilde{\mathbf{G}}$.

(a) If Property (i), resp. (ii), holds for both $U_{1}$ and $U_{2}$ then it holds for $U_{1} \otimes U_{2}$.

(b) If Property $(i)$ holds for both $U_{1}$ and $U_{2}$ and Property (ii) holds for $U_{1} \otimes U_{2}$, then Property (ii) holds for both $U_{1}$ and $U_{2}$.

(c) If $U=\int{ }^{\oplus} U(\lambda) d m(\lambda)$ is a direct integral decomposition of $U$, then Property (i), resp. (ii), holds for $U$ iff it holds for $U(\lambda)$, for $m$-almost all $\lambda$.

Proof. Part $(a)$ and $(c)$ are immediate by Lemma 1.2 since one can check Property (1.1) on natural dense sets.

If Property $(i i)$ holds for $U_{1} \otimes U_{2}$ then it holds for $U_{1}$ and $U_{2}$ up to a constant, namely, considering for example the representation $U_{1}$, there exists a phase $z(t)$ such that $T_{t} \subset z(t) e^{-i t H}$ and $T_{t}$ is densely defined. Equivalently $e^{i t H} e^{-\frac{1}{2} K}$ is densely defined and extended by $z(t) e^{-\frac{1}{2} K} e^{-1 t H}$ and this implies that $z(t)$ is a one-dimensional character.

Of course $z(\cdot)$ remains unchanged if we replace $\mu$ and $\nu$ by a pair of conjugate one-parameter subgroups. As in the proof of Lemma 1.2 we may thus replace $\mu(t)$ by $\mu(-t)$ and thus $z(t)$ by $z(-t)$, hence $z(t)=$ $z(-t)=1$ and the proof is complete.

We need now to verify Property (ii) in some specific representation. To this end recall that [43], if $W_{i}$ is the wedge in the 3-dimensional space-time along the axis $x_{i}, i=1,2$, and $\Lambda_{i}(t)$ is the associated one-parameter group of pure Lorentz transformations (see Sect. 2), there is an isomorphism of PSL(2, R) with the $2+1$-dimensional Lorentz group $\mathcal{L}_{+}^{\uparrow}(3)$ determined by

$$
\mu(t) \rightarrow \Lambda_{1}(t) \text { and } \quad \nu(t) \rightarrow \Lambda_{2}(t) .
$$

Accordingly, we shall identify $\mathbf{G}$ with a subgroup of the 2+1-dimensional Poincaré group $\mathcal{P}_{+}^{\uparrow}(3)$.

1.4. Lemma. Let $V \equiv V_{m, 0}$ be the positive energy representation of $\mathcal{P}_{+}^{\uparrow}(3)$ of spin 0 and mass $m>0$. Then Property (ii) holds for the restriction $\left.U \equiv V\right|_{\mathbf{G}}$ of $V$ to $\mathbf{G}$.

Proof. As is known, $V$ extends to a (anti-)representation of the proper Poincaré group $\mathcal{P}_{+}(3)$, namely there exists a anti-unitary involution $\Theta$, on the same Hilbert space, that commutes with $U$ and implements the change of sign on the translation operators in any space-time direction.

By the one-particle version of the Bisognano Wichmann theorem (which follows of course from the Bisognano-Wichmann theorem in the free field setting, see [30] for a short direct verification of this special case) we may identify the rescaled boost transformations with the modular group of the real Hilbert subspace of the one-particle Hilbert space associated to the corresponding wedge region. Then Property (1.1) holds because it is equivalent to the commutativity of $\Theta$ with the boosts (see Proposition 2.6).

1.5. Remark. The proof of Lemma 1.4 makes use of a one-particle version of the Bisognano-Wichmann theorem; as we mentioned this can be proved directly by mimicking the proof of the Bisognano-Wichmann 
in this special case. Since this quick verification requires an analytic continuation argument that does not fit with the spirit of this paper, we sketch here an algebraic derivation of Lemma 1.4. To begin with note that Lemma 1.4 would hold if $V$ were the irreducible positive-energy massless representation of $\mathcal{P}_{+}^{\uparrow}(3)$ with helicity 0 . Indeed in this case $V$ extends to a representation of the conformal group and the algebraic argument in [8] applies. Now $V \otimes V$ has a direct integral decomposition into irreducible representations where massive representations occur and thus Lemma 1.2 implies Lemma 1.4 for some $m>0$. However the representation $\left.U \equiv V\right|_{\mathbf{G}}$ in Lemma 1.4 does not depend on $m>0$ up to unitary equivalence by the following proposition, hence Lemma 1.4 holds for all $m>0$.

1.6. Proposition. The representation $\left.U \equiv V\right|_{\mathbf{G}}$ in Lemma 1.4 is equivalent to the quasi-regular representation of $\mathbf{G}$ corresponding to the rotation subgroup $\mathbf{K} \equiv\left\{\left(\begin{array}{cc}\cos \theta & \sin \theta \\ -\sin \theta & \cos \theta\end{array}\right), 0 \leq \theta<2 \pi\right\} /\{1,-1\}$, namely $U$ is the representation of $\mathbf{G}$ induced by the identity representation of $\mathbf{K}$. The representation $U \otimes U$ is equivalent to an infinite multiple of the regular representation $\lambda$ of $\mathbf{G}$

$$
U \otimes U=\infty \cdot \lambda
$$

Proof. The $m>0$ hyperboloid $H_{m}=\left\{\vec{x} \in \mathbf{R}^{3} / x_{0}^{2}-x_{1}^{2}-x_{2}^{2}=m^{2}, x_{0}>0\right\}$ is a homogeneous space for $\mathbf{G}$ whose stability subgroup at the point $(m, 0,0)$ is $\mathbf{K}$. $U$ is the corresponding representation on $L^{2}\left(H_{m}, \mu_{m}\right)$, with $\mu_{m}$ the Lorentz invariant measure on $H_{m}$, and this is by definition the quasi-regular represention with respect to $\mathbf{K}$. The last statement is a consequence of the Mackey tensor product theorem for induced representations, see [32, Theorem 2 and Example 5].

Proof of of Theorem 1.1. (a) Property ( (ii) for $\lambda$ follows by Lemma 1.2 and Proposition 1.6. Here is an alternative verification of this fact. By Proposition 1.6, taking tensor products and making use of Corollary 1.3, we check that Property (1.1) is valid for the irreducible representation $V_{m, s}$ of $\mathcal{P}_{+}^{\uparrow}(3)$ of any mass $m>0$ and any integral $\operatorname{spin} s$. Now $\left.U_{m, s} \equiv V_{m, s}\right|_{\mathbf{G}}$ is the induced representation

$$
U_{m, s}=\operatorname{Ind}_{\mathbf{K}}^{\mathbf{G}}\left(\chi_{s}\right)
$$

where $\chi_{s}$ is character of $\mathbf{K} \simeq \mathbf{T}$ associated with the integer $s$. By inducing at stages one has

$$
\lambda=\operatorname{Ind}_{\{1\}}^{\mathbf{G}}(i d)=\operatorname{Ind}_{\mathbf{K}}^{\mathbf{G}}\left(\lambda_{\mathbf{K}}\right)
$$

where $\lambda_{\mathbf{K}}=\operatorname{Ind}_{\{1\}}^{\mathbf{K}}(i d)$ is the regular representation of $\mathbf{K}$, hence

$$
\lambda=\operatorname{Ind}_{\mathbf{K}}^{\mathbf{G}}\left(\lambda_{\mathbf{K}}\right)=\operatorname{Ind}_{\mathbf{K}}^{\mathbf{G}}\left(\bigoplus_{s=-\infty}^{\infty} \chi_{s}\right)=\bigoplus_{s=-\infty}^{\infty} \operatorname{Ind}_{\mathbf{K}}^{\mathbf{G}}\left(\chi_{s}\right)=\bigoplus_{s=-\infty}^{\infty} U_{m, s}
$$

and the statement follows by Corollary 1.4 .

(b) Let $U$ enjoy Property $(i i)$. If $U$ is a general representation of $\widetilde{\mathbf{G}}$, then it determines a projective representation of $\mathbf{G}$, namely $U(l(\cdot))$ with $l$ a Borel section for the quotient map of $\widetilde{\mathbf{G}}$ modulo $\mathbf{G}$. The tensor product $U \otimes \bar{U}$ of $U$ with the conjugate representation is a true representation of $\mathbf{G}$, hence Property $(i i)$ holds for $U \otimes \bar{U}$. Then, by Lemma 1.2, Property (ii) holds for $U$. 


\section{PCT, Spin and Statistics on the Field Algebras}

In this section we consider a pre-cosheaf $\mathcal{O} \rightarrow \mathcal{F}(\mathcal{O})$ of von Neumann algebras acting on a Hilbert space $\mathcal{H}$, where $\mathcal{O}$ is any open region of the 4 -dimensional Minkowski space $M$. We assume the following properties:

(1) Reeh-Schlieder property for space-like cones: there is a vector $\Omega \in \mathcal{H}$ (vacuum) which is cyclic for the algebras associated with all space-like cones.

(2) Normal commutation relations: there is a vacuum-preserving self-adjoint unitary $\Gamma$ (statistics operator) that implements an automorphism on every local von Neumann algebra and the normal commutation relations between Bose and Fermi fields hold, i.e. setting

$$
\mathcal{F}_{ \pm}(\mathcal{O}):=\{A \in \mathcal{F}(\mathcal{O}): \Gamma A \Gamma= \pm A\}
$$

we have that if $\mathcal{O}_{1}$ and $\mathcal{O}_{2}$ are space-like separated then $\mathcal{F}_{+}\left(\mathcal{O}_{1}\right)$ commutes with $\mathcal{F}\left(\mathcal{O}_{2}\right)$ and $\mathcal{F}_{-}\left(\mathcal{O}_{1}\right)$ anticommutes with $\mathcal{F}_{-}\left(\mathcal{O}_{2}\right)$.

(3) Modular covariance property with respect to the vacuum vector $\Omega$ (cf. Definition 2.3).

2.1 Proposition. (Twisted Locality). Let $Z$ be the unitary operator defined by

$$
Z=\frac{I+i \Gamma}{1+i}
$$

Then

$$
Z \mathcal{F}(\mathcal{O}) Z^{*} \subset \mathcal{F}\left(\mathcal{O}^{\prime}\right)^{\prime}
$$

Proof. A direct computation shows that

$$
\begin{array}{cc}
Z B Z^{*}=B, & B \in \mathcal{F}_{+} \\
Z F Z^{*}=i \Gamma F & F \in \mathcal{F}_{-}
\end{array}
$$

Hence, if $\mathcal{O}_{1}$ and $\mathcal{O}_{2}$ are space-like separated and $F_{j} \in \mathcal{F}_{-}\left(\mathcal{O}_{j}\right), j=1,2$ we have

$$
\left[Z F_{1} Z^{*}, F_{2}\right]=i \Gamma\left(F_{1} F_{2}+F_{2} F_{1}\right)=0
$$

and the thesis holds.

We recall that a wedge region is any Poincaré transformed of the region $W_{1}:=\left\{\vec{x} \in \mathbf{R}^{n}:\left|x_{0}\right|<x_{1}\right\}$. The boosts preserving $W_{1}$ are the elements of the one-parameter subgroup $\Lambda_{W_{1}}(t)$ of $\mathcal{P}_{+}^{\uparrow}$ which acts on the coordinates $x_{0}, x_{1}$ via the matrices

$$
\left(\begin{array}{cc}
\cosh 2 \pi t & -\sinh 2 \pi t \\
-\sinh 2 \pi t & \cosh 2 \pi t
\end{array}\right)
$$

and leaves the other coordinates unchanged. The boosts $\Lambda_{W}(t)$ for any wedge $W$ are defined by Poincaré conjugation. We denote by $\mathcal{W}$ the family of all wedges in $M$.

By twisted locality and the Reeh-Schlieder assumption the vacuum is cyclic and separating for the algebras associated with all regions $\mathcal{B}$ such that both $\mathcal{B}$ and $\mathcal{B}^{\prime}$ contain some space-like cone. In particular, this property holds for any wedge region $W$, therefore, by Tomita-Takesaki theory, the modular operators $\Delta_{W}$ and $J_{W}$ associated with $(\mathcal{F}(W), \Omega)$ are defined for each region $W \in \mathcal{W}$.

2.2. Lemma. The following commutation relations hold for all $W \in \mathcal{W}$ :

$$
\begin{aligned}
& {\left[Z, \Delta_{W}^{i t}\right]=0} \\
& J_{W} Z J_{W}=Z^{*} .
\end{aligned}
$$


Proof. Since $\Gamma$ preserves the vacuum and maps $\mathcal{F}(W)$ in itself, it commutes with the modular operators. Then the relations follow by the definition of $Z$.

2.3. Definition. The pre-cosheaf $\mathcal{O} \rightarrow \mathcal{F}(\mathcal{O})$, with the Reeh-Schlieder property with respect to $\Omega$ as above, satisfies modular covariance if, for all regions $\mathcal{O}$,

$$
\Delta_{W}^{i t} \mathcal{F}(\mathcal{O}) \Delta_{W}^{-i t}=\mathcal{F}\left(\Lambda_{W}(t) \mathcal{O}\right), \quad W \in \mathcal{W}
$$

2.4. Proposition. Let $\mathcal{O} \rightarrow \mathcal{F}(\mathcal{O})$ be a modular covariant pre-cosheaf of von Neumann algebras satisfying the Reeh-Schlieder property and normal commutation relations. Then the modular unitaries associated to the wedges determine a positive energy unitary representation $U$ of the covering group $\widetilde{\mathcal{P}}_{+}^{\uparrow}$ of the Poincaré group such that

$$
U(g) \mathcal{F}(\mathcal{O}) U(g)^{*}=\mathcal{F}(\sigma(g) \mathcal{O}) \quad g \in \widetilde{\mathcal{P}}_{+}^{\uparrow}, \mathcal{O} \subset M
$$

where $\sigma: \widetilde{\mathcal{P}}_{+}^{\uparrow} \rightarrow \mathcal{P}_{+}^{\uparrow}$ is the covering map, and

$$
\Delta_{W}^{i t}=U\left(\tilde{\Lambda}_{W}(t)\right), \quad W \in \mathcal{W}
$$

where $\tilde{\Lambda}_{W}(t)$ denotes the lifting of $\Lambda_{W}(t)$ in $\widetilde{\mathcal{P}}_{+}^{\uparrow}$.

Before proving Proposition 2.4 we observe that twisted essential duality holds if modular covariance is assumed.

2.5 Proposition. (Twisted essential duality). If the pre-cosheaf $\mathcal{F}$ satisfies modular covariance, then

$$
Z \mathcal{F}(W) Z^{*}=\mathcal{F}\left(W^{\prime}\right)^{\prime}
$$

As a consequence,

$$
\Delta_{W}^{i t}=\Delta_{W^{\prime}}^{-i t}
$$

Proof. By modular covariance, twisted locality and Lemma $2.2, Z \mathcal{F}(W) Z^{*}$ is a globally invariant subalgebra of $\mathcal{F}\left(W^{\prime}\right)^{\prime}$ under the action of the modular group $\Delta_{W^{\prime}}^{i t}$ for which the vacuum is cyclic, therefore it coincides with $\mathcal{F}\left(W^{\prime}\right)^{\prime}$. The last equality follows by Lemma 2.2 .

Proof of of Proposition 2.4. The steps of the proof are essentially the same as in the proof of Theorem 2.3 in [9], but Lemma 2.5 [9] should be reconsidered because we do not assume additivity, and Fermi statistics is allowed. Indeed the first part, in which it is shown that the set $H=\left\{g \in \mathcal{P}_{+}^{\uparrow}: \mathcal{F}(g \mathcal{O})=\mathcal{F}(\mathcal{O})\right.$, $\mathcal{O} \subset M\}$ is a normal subgroup of $\mathcal{P}_{+}^{\uparrow}$, still holds. Therefore, with the same argument as in Lemma 2.6 [9], we get a central extension of $\mathcal{P}_{+}^{\uparrow} / H$.

When $H$ is $\{1\}$ or the translation subgroup, the triviality of the central extensions for $\mathcal{P}_{+}^{\uparrow}$ and for $\mathcal{L}_{+}^{\uparrow}$ concludes the theorem. When $H=\mathcal{P}_{+}^{\uparrow}$ then the algebras associated to all wedges coincide and, in particular, $\mathcal{F}(W)=\mathcal{F}\left(W^{\prime}\right)$. In this case $\mathcal{F}_{+}(W)$ is abelian and two Fermi fields localized in $W$ anticommute. Then if $A \in \mathcal{F}_{-}(W)$ we have $A^{*} A+A A^{*}=0$ which implies $\mathcal{F}_{-}(W)=0$. As a consequence $\mathcal{F}(W)$ is abelian for any wedge $W$ and the representation of $\widetilde{\mathcal{P}}_{+}^{\uparrow}$ implemented by the modular unitaries is the trivial representation.

We notice that in the proof of the preceding theorem the Reeh-Schlieder property was needed only for wedge regions. When this property does not hold for double cones it is possible to construct theories for which the group $H$ in the proof is the translation group, namely the translations act identically on the pre-cosheaf (see [23]).

Our next step is to show that, as a consequence of Theorem 1.1, the representation $U$ extends to (some covering of) the proper Poincaré group $\mathcal{P}_{+}$.

2.6. Proposition. Let $W_{1}, W_{2}$ be orthogonal wedges, and let us denote by $\Delta_{i}, J_{i}, i=1,2$ the modular operators of $\left(\mathcal{F}\left(W_{i}\right), \Omega\right)$. Then the following relation holds

$$
\Delta_{1}^{1 / 2} \Delta_{2}^{i t} \Delta_{1}^{-1 / 2} \subset J_{1} \Delta_{2}^{i t} J_{1}
$$


and the left hand side is densely defined.

Proof. We may suppose that $W_{i}:=\left\{\vec{x} \in \mathbf{R}^{n}:\left|x_{0}\right|<x_{i}\right\}, i=1,2$. With the same arguments of Lemma 1.2, it is enough to show that, for each $t \in \mathbf{R}$, there exists a dense set $\mathcal{D}_{t}$ such that

$$
\Delta_{1}^{1 / 2} \Delta_{2}^{i t} \Delta_{1}^{-1 / 2} \xi=J_{1} \Delta_{2}^{i t} J_{1} \xi \quad \xi \in \mathcal{D}_{t}
$$

We claim that we may take

$$
\mathcal{D}_{t}=\left\{J_{1} A \Omega: A \in \mathcal{F}\left(W_{1} \cap \Lambda_{2}(-t) W_{1}\right)\right\} .
$$

First we observe that Relation (2.6) holds on $\mathcal{D}_{t}$. Indeed, for $\xi=J_{1} A \Omega \in \mathcal{D}_{t}$,

$$
\begin{aligned}
J_{1} \Delta_{2}^{i t} J_{1} \xi & =J_{1} \Delta_{2}^{i t} A \Omega=J_{1}\left(\Delta_{2}^{i t} A \Delta_{2}^{-i t}\right) \Omega=\Delta_{1}^{1 / 2} \Delta_{2}^{i t} A^{*} \Omega \\
& =\Delta_{1}^{1 / 2} \Delta_{2}^{i t} S_{1} J_{1} \xi=\Delta_{1}^{1 / 2} \Delta_{2}^{i t} \Delta_{1}^{-1 / 2} \xi
\end{aligned}
$$

Now, by the Reeh-Schlieder property for space-like cones, it is enough to show that $\Lambda_{2}(-t) W_{1} \cap W_{1}$ contains a space-like cone in order to prove that $\mathcal{D}_{t}$ is dense. Indeed the space-like cone

$$
\mathcal{C}_{t}=\left\{\vec{x} \in M: \sqrt{x_{0}^{2}+x_{2}^{2}}<\frac{x_{1}}{\sqrt{2} \cosh 2 \pi t}\right\}
$$

is obviously contained in $W_{1}$ and, if $\vec{y}=\Lambda_{2}(t) \vec{x}, \vec{x} \in \mathcal{C}_{t}$, then

$$
\left|y_{0}\right|=\left|\cosh 2 \pi t x_{0}-\sinh 2 \pi t x_{2}\right| \leq \sqrt{2} \cosh 2 \pi t \sqrt{x_{0}^{2}+x_{2}^{2}}<x_{1}
$$

namely $\Lambda_{2}(t) \mathcal{C}_{t} \subset W_{1}$

2.7. Corollary. Let $W_{1}, W_{2}$ be orthogonal wedges, and let us denote by $\Delta_{i}, J_{i}, i=1,2$ the modular operators of $\left(\mathcal{F}\left(W_{i}\right), \Omega\right)$. Then, for each $t \in \mathbf{R}$, the following relation holds:

$$
J_{1} \Delta_{2}^{i t} J_{1}=\Delta_{2}^{-i t}
$$

Proof. By Proposition 2.6 it is enough to show that, for each $t \in \mathbf{R}$,

$$
\Delta_{1}^{1 / 2} \Delta_{2}^{i t} \Delta_{1}^{-1 / 2} \subset \Delta_{2}^{-i t}
$$

Since the two wedges are orthogonal, the homomorphism $\varphi: \operatorname{SL}(2, \mathbf{R}) \rightarrow \widetilde{\mathcal{P}}_{+}^{\uparrow}$ determined by $\varphi(\mu(t))=\tilde{\Lambda}_{W_{1}}(t)$ and $\varphi(\nu(t))=\tilde{\Lambda}_{W_{2}}(t)$ is injective. Then we apply Theorem 1.1 and the proof is complete.

Let us consider the automorphism $\alpha$ of the Poincaré group given by $\alpha(g)=I_{1} g I_{1}$, where $I_{1}$ is the diagonal matrix which changes the sign of the $x_{0}$ and $x_{1}$ components and leave the other coordinates fixed. The proper Poincaré group $\mathcal{P}_{+}$is generated by $I_{1}$ and $\mathcal{P}_{+}^{\uparrow}$, as a consequence it may be seen as a semidirect product:

$$
\mathcal{P}_{+}=\mathcal{P}_{+}^{\uparrow} \times{ }_{\alpha} \mathbf{Z}_{2} \text {. }
$$

The action $\alpha$ gives rise to an action on the Lie algebra of $\mathcal{P}_{+}^{\uparrow}$ and therefore to an action on the universal covering $\widetilde{\mathcal{P}}_{+}^{\uparrow}$ of $\mathcal{P}_{+}^{\uparrow}$.

Since the center of $\widetilde{\mathcal{P}}_{+}^{\uparrow}$ has order $2, \alpha$ is trivial on the center.

Now let us denote by $\widetilde{\mathcal{P}}_{+}$the semidirect product of the universal covering of $\mathcal{P}_{+}^{\uparrow}$ with $\mathbf{Z}_{2}$ via $\alpha$. It follows that $\widetilde{\mathcal{P}}_{+}$is a central extension of the proper Poincaré group $\mathcal{P}_{+}$via the homomorphism $\sigma$ extended by $\sigma\left(\tilde{I}_{1}\right)=I_{1}$, where $\tilde{I}_{1}$ denotes the non-trivial element in the $\mathbf{Z}_{2}$ component of $\widetilde{\mathcal{P}}_{+}$.

2.8. Proposition. The representation $U$ extends to a (anti-)unitary representation (still denoted by $U$ ) of the group $\widetilde{\mathcal{P}}_{+}$with $U\left(\tilde{I}_{1}\right)=Z J_{1}$.

Proof. We only have to show that the following relation holds:

$$
Z J_{1} U(g)\left(Z J_{1}\right)^{*}=U(\alpha(g)), \quad g \in \widetilde{\mathcal{P}}_{+}^{\uparrow} .
$$


Relation (2.9) holds for $\tilde{\Lambda}_{W_{1}}(t)$ by Tomita-Takesaki theory, for the translations by Borchers theorem [6] and for $\tilde{\Lambda}_{W}(t)$ when $W$ is a wedge orthogonal to $W_{1}$ by Lemma 2.2 and Proposition 2.5. Since the lifting of the boosts associated with a maximal set of orthogonal wedges and the translations generate $\widetilde{\mathcal{P}}_{+}^{\uparrow}$, we get the thesis.

We may associate a dual pre-cosheaf $\mathcal{F}^{d}$ to the given pre-cosheaf $\mathcal{F}$. If $\mathcal{O}$ is a convex causally complete region the algebra $\mathcal{F}^{d}(\mathcal{O})$ is defined by

$$
\mathcal{F}^{d}(\mathcal{O}) \equiv \bigcap_{W \supset \mathcal{O}} \mathcal{F}(W)
$$

where $W$ is a wedge. For any more general region $\mathcal{B}$ we set

$$
\mathcal{F}^{d}(\mathcal{B}) \equiv\left(\bigcup_{\mathcal{O} \subset \mathcal{B}} \mathcal{F}(\mathcal{O})\right)^{\prime \prime},
$$

where $\mathcal{O}$ is a convex causally complete region. It is easy to see that $\mathcal{F}^{d}$ is a pre-cosheaf satisfying ReehSchlieder property, locality and duality for convex causally complete regions.

2.9. Proposition. The representation $U$ of $\widetilde{\mathcal{P}}_{+}$implements a covariant action on the wedges, i.e.

$$
U(g) \mathcal{F}(W) U(g)^{*}=\mathcal{F}(g W), \quad \forall g \in \widetilde{\mathcal{P}}_{+}, W \in \mathcal{W} .
$$

As a consequence, the action is covariant on the dual pre-cosheaf $\mathcal{O} \rightarrow \mathcal{F}^{d}(\mathcal{O})$.

Proof. By Proposition 2.4, we have to prove covariance only for the element $Z J_{1}$. For each $W \in \mathcal{W}$ we may find a $g \in \widetilde{\mathcal{P}}_{+}$such that $W=\sigma(g) W_{1}$. Then

$$
\begin{aligned}
Z J_{1} \mathcal{F}(W)\left(Z J_{1}\right)^{*} & =Z J_{1} U(g) \mathcal{F}\left(W_{1}\right)\left(Z J_{1} U(g)\right)^{*} \\
& =Z J_{1} U(g) Z J_{1} \mathcal{F}\left(I_{1} W_{1}\right)\left(Z J_{1} U(g) Z J_{1}\right)^{*} \\
& =U(\alpha(g)) \mathcal{F}\left(-W_{1}\right) U(\alpha(g))^{*} \\
& =\mathcal{F}\left(I_{1} W\right) .
\end{aligned}
$$

Since the dual pre-cosheaf $\mathcal{F}^{d}$ is described in terms of the restriction of $\mathcal{F}$ to the wedges, the second part of the statement follows.

2.10 Theorem. (PCT). Let $\mathcal{O} \rightarrow \mathcal{F}(\mathcal{O})$ be a modular covariant pre-cosheaf satisfying Reeh-Schlieder property and normal commutation relations as above. Then there exists an antiunitary operator $\Theta$ which implements the PCT symmetry on the dual pre-cosheaf, i.e.

$$
\Theta \mathcal{F}^{d}(\mathcal{O}) \Theta=\mathcal{F}^{d}(-\mathcal{O}) .
$$

Proof. Since the dimension of the space-time is even, the transformation which changes the sign of all coordinates belongs to the proper Poincaré group $\mathcal{P}_{+}$. Then we choose a preimage $\theta \in \widetilde{\mathcal{P}}_{+}$via $\sigma$ of this element and set $\Theta:=U(\theta)$. Obviously $\Theta$ verifies Eq. (2.10) and is anti-unitary.

We remark that Proposition 2.9 is an abstract form of the PCT theorem and is indeed what we can prove in an odd dimensional Minkowski space, where the global space-time inversion does not belong to the proper Poincaré group.

2.11 Theorem. (Spin and Statistics). Let $\mathcal{O} \rightarrow \mathcal{F}(\mathcal{O})$ be a modular covariant pre-cosheaf of field algebras as in Theorem 2.10. Then

$$
\Gamma=U(2 \pi) .
$$

Proof. Let $W_{2}$ be the wedge along the axis $x_{2}$. It is well known that the generator of the rotations $r(\vartheta)$ on the $x_{1}-x_{2}$ plane and such that $r(\pi / 2) W_{1}=W_{2}$ is a multiple of the commutator between the generators 
of $\Lambda_{1}(t)$ and $\Lambda_{2}(t)$. Then, by definition, $\alpha(\tilde{r}(\vartheta))=\tilde{r}(-\vartheta)$, where $\tilde{r}$ denotes the lifting of $r$ in $\widetilde{\mathcal{P}}_{+}^{\uparrow}$. Therefore we have

$$
Z J_{1} Z J_{2}=Z J_{1} U\left(\tilde{r}\left(\frac{\pi}{2}\right)\right) Z J_{1} U\left(\tilde{r}\left(-\frac{\pi}{2}\right)\right)=U\left(\alpha\left(\tilde{r}\left(\frac{\pi}{2}\right)\right)\right) U\left(\tilde{r}\left(-\frac{\pi}{2}\right)\right)=U(\tilde{r}(-\pi)) .
$$

On the other hand, since $I_{1} W_{2}=W_{2}$, Proposition 2.9 shows that $Z J_{1}$ leaves $\mathcal{F}\left(W_{2}\right)$ globally stable, hence it commutes with $J_{2}$. Therefore

$$
Z J_{1} Z J_{2}=Z J_{1} J_{2} Z^{*}=J_{2} Z J_{1} Z^{*}=Z^{*} J_{2} Z J_{1}=\Gamma Z J_{2} Z J_{1}
$$

Finally,

$$
U(2 \pi)=\left(Z J_{1} Z J_{2}\right)^{2}=\left(\Gamma Z J_{2} Z J_{1}\right)\left(Z J_{1} Z J_{2}\right)=\Gamma
$$

\section{PCT and Spin and Statistics Theorems for Superselection Sectors with Finite Statistics}

In this section we shall prove the Spin and Statistics theorem in the framework of local observable algebras [24], i.e. we shall prove that a para-Bose (resp. para-Fermi) superselection sector with finite statistics is covariant with respect to the group $\mathcal{P}_{+}^{\uparrow}$ (resp. $\widetilde{\mathcal{P}}_{+}^{\uparrow}$ ).

For the sake of simplicity, in this section we shall make the usual assumption the von Neumann algebras associated with unbounded regions are generated by additivity by the ones associated with double cones within the region, so that the Doplicher-Roberts theorem on the reconstruction of the field algebras applies [18].

We shall consider morphisms of the local observable algebras localized in space-like cones in a 4dimensional space-time $[15,11]$. The same techniques would prove the Spin and Statistics theorem for sectors localized in space-like cones in the $n$-dimensional Minkowski space for any $n \geq 4$ and for sectors localized in double-cones if $n=3$. We omit here the treatment of these cases in view of a more general intrinsic proof [23], valid also in the low-dimensional case, that will be carried on in the setting of the of pre-cosheaves of von Neumann algebras on wedge regions.

In the following we consider a net of local observable von Neumann algebras [27], i.e. a map

$$
\mathcal{A}: \mathcal{O} \rightarrow \mathcal{A}(\mathcal{O}), \quad \mathcal{O} \in \mathcal{K}
$$

where $\mathcal{K}$ is the family of the double cones in the 4-dimensional Minkowski space $M$, such that

$$
\begin{aligned}
\mathcal{O}_{1} \subset \mathcal{O}_{2} & \Rightarrow \mathcal{A}\left(\mathcal{O}_{1}\right) \subset \mathcal{A}\left(\mathcal{O}_{2}\right) \\
\mathcal{A}(\mathcal{O}) & \subset \mathcal{A}\left(\mathcal{O}^{\prime}\right)^{\prime}
\end{aligned}
$$

If $\mathcal{B}$ is an unbounded open region of $M$, we shall denote by $\mathcal{A}(\mathcal{B})$ the von Neumann algebra generated by the von Neumann algebras $\mathcal{A}(\mathcal{O}), \mathcal{O} \subset \mathcal{B}$, so that $\mathcal{A}$ extends to a pre-cosheaf of von Neumann algebras on more general regions. The local algebras are supposed to act on a separable Hilbert space $\mathcal{H}_{0}$, with a common cyclic vector $\Omega_{0}$.

The modular covariance property with respect to $\Omega_{0}$ is assumed. As a particular case of the analysis in the previous section, this property gives rise to a positive-energy representation of the Poincaré group which leaves $\Omega_{0}$ invariant. By locality $\Omega_{0}$ is cyclic and separating for the von Neumann algebras associated with all non-empty open regions whose complement have non-empty interior (Reeh-Schlieder property).

3.1. Proposition. $\mathcal{A}$ has a unique direct integral decomposition into irreducible modular covariant nets.

Proof. If $\xi \in \mathcal{H}_{0}$ is a $\Delta_{W}^{i t}$-invariant vector for some wedge $W$, then $\xi$ is also $\Delta_{W_{1}}^{i t}$-invariant for all other wedges $W_{1}$. This follows by the representation theory of $\mathrm{SL}(2, \mathbf{R})$, since the modular operators generate a representation of the Poincaré group. Therefore the centralizer $\mathcal{Z}$ of $\mathcal{A}(W)$ with respect to $\Omega_{0}$ does not 
depend on $W$. By locality $\mathcal{Z}$ is an abelian von Neumann algebra. The direct integral decomposition with respect to $\mathcal{Z}$ has then the desired properties. The uniqueness is clear since $\mathcal{Z}$ is canonically constructed.

Because of Proposition 3.1 we shall always assume in the following that the net is irreducible. We summarize now the results so far obtained in the following theorem.

3.2. Theorem. Let $\mathcal{O} \rightarrow \mathcal{A}(\mathcal{O})$ an irreducible local, modular covariant net of von Neumann algebras on $M$ as above. The extended pre-cosheaf satisfies essential duality, and there is a (unique) positive energy unitary representation $g \in \mathcal{P}_{+}^{\uparrow} \rightarrow U_{0}(g)$ with the following properties:

(a) $U_{0}(g) \mathcal{A}(\mathcal{O}) U_{0}(g)^{*}=\mathcal{A}(g \mathcal{O})$,

(b) $U_{0}\left(\Lambda_{W}(t)\right)=\Delta_{W}^{i t}, W \in \mathcal{W}$,

(c) $[\mathrm{PCT}$ Theorem] There is an antiunitary operator $\Theta$ (PCT operator) satisfying

$$
\begin{aligned}
\Theta \mathcal{A}(\mathcal{O}) \Theta & =\mathcal{A}(-\mathcal{O}), \quad \mathcal{O} \in \mathcal{K} \\
{[\operatorname{ad} \Theta \cdot \rho] } & =[\bar{\rho}],
\end{aligned}
$$

where $\rho$ is a morphism localized in a space-like cone, and the PCT operator is given by $\Theta=J_{W} R_{W}$ where $W$ is any wedge whose edge contains the origin and $R_{W}$ is the space reflection w.r.t. all directions contained in the edge of $W$.

Proof. Since the net $\mathcal{A}$ may be considered as a purely Bose pre-cosheaf of field algebras (cf. Sect. 2), the thesis follows by Theorems 2.9 and 2.10 and by [22].

The previous theorem shows in particular that the Spin and Statistics theorem holds in the vacuum sector, solving a problem that remained open in [9].

We recall now that a localized representation of the quasi-local $\mathrm{C}^{*}$-algebra

$$
\mathcal{A}:=\left(\bigcup_{\mathcal{O} \in \mathcal{K}} \mathcal{A}(\mathcal{O})\right)^{-}
$$

(norm closure) is a representation which is equivalent to the vacuum representation when restricted to the space-like complement of any space-like cone. Such a representation is equivalent a localized transportable morphism, that is to say a morphism $\rho$ of $\mathcal{A}$ into $B\left(\mathcal{H}_{0}\right)$ which is localized in a given space-like cone $\mathcal{S}$, i.e. $\rho(x)=x$ for each $x \in \mathcal{A}\left(\mathcal{S}^{\prime}\right)$.

A unitary equivalence class of localized representations is called a superselection sector.

A localized morphism $\rho$ is called Poincaré covariant if there exists a unitary representation $U_{\rho}$ of the covering of the Poincaré group $\widetilde{\mathcal{P}}_{+}^{\uparrow}$ verifying

$$
U_{\rho}(g) \rho(x) U_{\rho}(g)^{*}=\rho\left(U_{0}(\sigma(g)) x U_{0}^{*}(\sigma(g))\right), \quad g \in \widetilde{\mathcal{P}}_{+}^{\uparrow}, x \in \mathcal{A} .
$$

We recall that when the net satisfies a mild condition called regularity, all localized transportable morphisms with finite statistics are Poincaré covariant [22].

As already mentioned, a sector carries a projective representation of the Poincaré group, i.e. $U_{\rho}(2 \pi)$ is not necessarily the identity. If $\rho$ is irreducible $U_{\rho}(2 \pi)= \pm 1$.

If an irreducible morphism $\rho$ with finite statistics is localized in a double cone, Doplicher, Haag and Roberts [15] showed that the statistical behaviour of $\rho$ is described by the statistical parameter $\lambda_{\rho}$, an analysis extended to topological charges by Buchholz and Fredenhagen [11]. As explained in the Introduction, the spin-statistics relation takes the form of an equality of complex numbers, i.e. $U_{\rho}(2 \pi)=\operatorname{sign}\left(\lambda_{\rho}\right)$.

3.3 Theorem. (Spin and Statistics). Let $\mathcal{O} \rightarrow \mathcal{A}(\mathcal{O})$ be an irreducible, local, modular covariant net of von Neumann algebras on the Minkowski space and let $\rho$ be an irreducible, covariant, localized morphism with finite statistics. Then

$$
U_{\rho}(2 \pi)=\operatorname{sign}\left(\lambda_{\rho}\right) .
$$


By the Doplicher-Roberts theorem [18], the superselection structure is described by a field net $\mathcal{O} \rightarrow \mathcal{F}(\mathcal{O})$ of von Neumann algebras satisfying normal commutation relations and by a vacuum-preserving unitary representation $V$ of a compact group $G$ (the gauge group) on a Hilbert space $\mathcal{H}$, that implements automorphisms on any $\mathcal{F}(\mathcal{O})$.

There is a canonical net isomorphism $\pi: \mathcal{A}(\mathcal{O}) \rightarrow \mathcal{F}^{G}(\mathcal{O})$ where $\mathcal{F}^{G}(\mathcal{O})$ is the fixed-point algebra under the gauge action, and the covariance property of the sectors gives a uniquely determined representation $U$ of $\widetilde{\mathcal{P}}_{+}^{\uparrow}$ on $\mathcal{H}$ which commutes with the gauge group representation and restricts to $U_{0}$ on the vacuum Hilbert space $\mathcal{H}_{0}$.

3.4. Lemma. The representation $U$ satisfies

$$
U\left(\Lambda_{W}(t)\right)=\Delta_{W}^{i t}, \quad W \in \mathcal{W}, t \in \mathbf{R} .
$$

Proof. With $W$ a wedge region, denote by $\Delta_{W}$ the modular operator associated with $\mathcal{F}(W)$. Observe that $c_{W}(t):=U\left(\Lambda_{W}(t)\right) \Delta_{W}^{-i t}$ implements an automorphism of the algebra $\mathcal{F}(W)$ leaving $\mathcal{F}^{G}(W)$ pointwise fixed by Theorem 3.1(b). The translations along $W$ commute with $c_{W}(t)$, since they commute both with $U\left(\Lambda_{W}(t)\right)$ and $\Delta_{W}^{i t}$, and act ergodically on $\mathcal{F}(W)$ by the irreducibility of the net. By the Galois correspondence [1], it follows that $c_{W}(\cdot)$ is a one-parameter subgroup of $V(G)$.

We show now that $c_{W}(t)=I$. Indeed, on the one hand, by twisted duality (Proposition 2.5)

$$
c_{W^{\prime}}(t)=c_{W}(-t), \quad W \in \mathcal{W}, t \in \mathbf{R} .
$$

On the other hand $c_{g W}(t)=U(g) c_{W}(t) U(g)^{*}=c_{W}(t)$ because the gauge group action commutes with the representation $U$ of $\widetilde{\mathcal{P}}_{+}^{\uparrow}$, which means that $c_{W}(t)$ does not depend by $W$. Then $c_{W}(t)=I$, i.e. $U\left(\Lambda_{W}(t)\right)=\Delta_{W}^{i t}$.

Proof of of Theorem 3.3. $\quad$ Each irreducible superselection sector $\rho$ is associated in a one-to-one correspondence with an irreducible representation $V_{\rho}$ of the gauge group $G$. The Hilbert space $\mathcal{H}$ decomposes into a direct sum

$$
\mathcal{H}=\bigoplus_{\rho} \mathcal{H}_{\rho}
$$

where $\mathcal{H}_{\rho}:=\mathcal{H}_{0} \otimes \mathbf{C}^{d(\rho)}$ and $d(\rho)=\left|\lambda_{\rho}\right|^{-1}=\operatorname{dim}\left(V_{\rho}\right)$ is the statistical dimension of $\rho$, so that the representation $\pi$ of the observable algebra $\mathcal{A}$ on $\mathcal{H}$, the representation $U$ of $\widetilde{\mathcal{P}}_{+}^{\uparrow}$ and the representation $V$ of the gauge group decompose accordingly as

$$
\begin{aligned}
\pi(x) & =\bigoplus_{\rho \in \hat{G}} \rho(x) \otimes I, \quad x \in \mathcal{A}, \\
U(g) & =\bigoplus_{\rho \in \hat{G}} U_{\rho}(g) \otimes I, \quad g \in \widetilde{\mathcal{P}}_{+}^{\uparrow}, \\
V(g) & =\bigoplus_{\rho \in \hat{G}} I \otimes V_{\rho}(g), \quad g \in G,
\end{aligned}
$$

where $U_{\rho}$ is the representation of $\widetilde{\mathcal{P}}_{+}^{\uparrow}$ in the sector $\rho$ as before.

In particular the statistics operator $\Gamma$ is constant on the direct summands $\mathcal{H}_{\rho}$, and we have

$$
\Gamma=\bigoplus_{\rho} \kappa_{\rho} I_{\mathcal{H}_{\rho}}
$$

where $\kappa_{\rho}=\operatorname{sign}\left(\lambda_{\rho}\right)$ is the statistics phase.

Then the Spin and Statistics theorem follows directly by Theorem 2.10.

Acknowledgements. We thank Klaus Fredenhagen for pointing out a gap in the proof of Theorem 1.1 of our original manuscript. 


\section{References}

[1] Araki, H., Haag, R., Kastler, D., Takesaki, M.: "Extensions of KMS states and chemical potential." Commun. Math. Phys. 53, 97-134 (1977)

[2] Bisognano, J., Wichmann, E.: "On the duality condition for a Hermitian scalar field." J. Math. Phys. 16, 985-1007 (1975)

[3] Bisognano, J., Wichmann, E.: "On the duality condition for quantum fields." J. Math. Phys. 17, 303-321 (1976)

[4] Bjorken, J.D., Drell, S.D.: Relativistic Quantum Fields. New York: McGraw-Hill, 1965

[5] Borchers, H.J.: "Local rings and the connection between spin and statistics." Commun. Math. Phys. 1, 281-307 (1965)

[6] Borchers, H.J.: "The CPT theorem in two-dimensional theories of local observables." Commun. Math. Phys. 143, 315 (1992)

[7] Borchers, H.J.: "On the converse of the Reeh-Schlieder theorem." Commun. Math. Phys. 10, 269-273 (1968)

[8] Brunetti, R., Guido, D., Longo, R.: "Modular structure and duality in conformal quantum field theory." Commun. Math. Phys. 156, 201-219 (1993)

[9] Brunetti, R., Guido, D., Longo, R.: "Group cohomology, modular theory and space-time symmetries." Rev. Math. Phys. 7, 57-71 (1994)

[10] Buchholz, D., Epstein, H.: "Spin and statistics of quantum topological charges." Fizika 3, 329-343 (1985)

[11] Buchholz, D., Fredenhagen, K.: "Locality and structure of particle states." Commun. Math. Phys. 84, 1-54 (1982)

[12] Buchholz, D., Summers, S.J.: "An algebraic characterization of vacuum states in Minkowski space." Commun. Math. Phys. 155, 442-458 (1993)

[13] Burgoyne, N.: "On the connection of spin with statistics." Nuovo Cimento 8, 807 (1958)

[14] Dell'Antonio, G.F.: "On the connection of spin with statistics." Ann. Phys. 16, 153 (1961)

[15] Doplicher, S., Haag, R., Roberts, J.E.: "Local observables and particle statistics I." Commun. Math. Phys. 23, 199-230 (1971)

[16] Doplicher, S., Haag, R., Roberts, J.E.: "Local observables and particle statistics II." Commun. Math. Phys. 35, 49-85 (1974)

[17] Doplicher, S., Longo, R.: "Standard and split inclusions of von Neumann algebras." Invent. Math. 73, 493-536 (1984)

[18] Doplicher, S., Roberts, J.E.: "Why there is a field algebra with a compact gauge group describing the superselection structure in particle physics." Commun. Math. Phys. 131, 51-107 (1990)

[19] Dyson, F.J.: "On the connection of weak local commutativity and regularity of Wightman functions." Phys. Rev. 110, 579 (1958)

[20] Fierz, M.: "Über die relativische Theorie kräftfreier Teilchen mit beliebigem spin." Helv. Phys. Acta 12, 3 (1939)

[21] Epstein, H.: "CTP invariance in a theory of local observables." J. Math. Phys. 8, 750 (1967)

[22] Guido, D., Longo, R.: "Relativistic invariance and charge conjugation in quantum field theory." Commun. Math. Phys. 148, 521-551 (1992)

[23] Guido, D., Longo, R.: "The Conformal Spin and Statistics theorem.". Preprint. Brunetti, R., Guido, D., Longo, R.: in preparation.

[24] Haag, R.: Local Quantum Physics. Berlin Heidelberg: Springer Verlag, 1992

[25] Haag, R., Hugenoltz, N.M., Winnink, M.: "On the equilibrium states in quantum statistical mechanics." Commun. Math. Phys. 5, 215 (1967)

[26] Hawking, S.W.: "Particle creation by black holes." Commun. Math. Phys. 43, 199 (1975)

[27] Haag, R., Kastler, D.: "An algebraic approach to Quantum Field Theory." J. Math. Phys. 5, 848-861 (1964)

[28] Jost, R.: The general theory of Quantized Fields. Providence RI: Am. Math. Soc., 1965

[29] Jost, R.: "Eine Bemerkung zu CTP Theorem." Helv. Phys. Acta 30, 409 (1957)

[30] Kay B.S., "A Uniqueness Result for Quasi-Free KMS States" Helv. Phys. Acta 58, 1017-1029 (1985)

[31] Kuckert, B.: "PCT und Kovarianz als modulare Strukturen." Diploma Thesis, Hamburg, in preparation 
[32] Lipsman, R.L.: "Group representations." Lect. Notes in Math. 388, New York-Heidelberg-Berlin: Springer Verlag, 1974

[33] Landau, L.: "On local functions of fields." Commun. Math. Phys. 39, 49-62 (1974)

[34] Longo, R.: "Index of subfactors and statistics of quantum fields." I Commun. Math. Phys. 126, 217-247 (1989); II 130, 285-309 (1990)

[35] Lüders, G.: "Vertaugschungsrelationen zwischen verschiedenen Feldern." Z. Naturforsch. 13a, 254 (1958)

[36] Lüders, G., Zumino, B.: "Connection between spin and statistics." Phys. Rev. 110, 1450 (1958)

[37] Pauli, W.: "On the connection between spin and statistics." Phys. Rev. 58, 716 (1940)

[38] Pauli, W.: "Exclusion principle, Lorentz group and reflection of space time and charge." In: Niels Bohr and the Development of Physics, W. Pauli (ed.) New York: Pergamon Press, 1955

[39] Schwinger, J.: "On the theory of quantized fields I." Phys. Rev. 82, 914 (1951)

[40] Sewell, G.L.: "Relativity of temperature and Hawking effect." Phys. Lett. 79A, 23 (1980)

[41] Strătilă, S., Zsido, L.: Lectures on von Neumann algebras. England: Abacus Press, 1979

[42] Streater, R.F.: Local fields with the wrong connection between Spin and Statistics. Commun. Math. Phys. 5, 88-96 (1967)

[43] Streater, R.F., Wightman, A.S.: PCT, Spin and Statistics, and all that. Reading (MA): Benjamin, 1964

[44] Takesaki, M.: "Tomita theory of modular Hilbert algebras." Lect. Notes in Math. 128, New YorkHeidelberg-Berlin: Springer Verlag, 1970

[45] Unruh, W.G.: "Notes on black hole evaporation." Phys. Rev. D14, 870 (1976)

[46] Wightman, A.S.: "Quantum field theory in terms of vacuum expectation values." Phys. Rev. 101, 860 (1956)

[47] Wiesbrock, H.V.: "A comment on a recent work of Borchers." Lett. Math. Phys. 25, 157-159 (1992)

[48] Yngvason, J.: "A note on essential duality." Lett. Math. Phys. 31, 127-141 (1994) 